\title{
Three Dimensional Vibration Analysis of Rectangular Plates with Undamaged and Damaged Boundaries by the Spectral Collocation Method
}

\author{
Ma'en S. Sari \\ Department of Mechanical Engineering, King Faisal University, Al-Hasa 31928, Saudi Arabia \\ Eric A. Butcher \\ Department of Mechanical and Aerospace Engineering, New Mexico State University, Las Cruces NM 88003, USA
}

(Received 14 May 2012; provisionally accepted 29 November 2012; accepted 13 February 2013)

\begin{abstract}
The three-dimensional free vibration analysis of isotropic rectangular elastic plates with standard undamaged or damaged boundaries is studied using the Chebyshev spectral collocation method. It was assumed that the plates had two free lateral surfaces and two opposite simply supported surfaces, while the other surfaces could be clamped, simply supported, free, or damaged clamped boundaries. In the present study the boundary conditions are coupled with the governing equation to obtain the equivalent algebraic eigenvalue problem in terms of the Chebyshev collocation grid. The results obtained indicate the behavior of the natural vibration frequencies with respect to the severity of the damaged boundary. This analysis can possibly lead to an efficient technique for damage detection of structures in which joint or boundary damage plays a significant role in the dynamic characteristics.
\end{abstract}

\section{INTRODUCTION}

Identifying and detecting damage is one of the most important engineering topics of recent decades. Damage is defined as a change in the system that affects its current or future performance. As far as damage accumulated in structural elements is concerned, it is usually estimated in terms of changes in overall elastic stiffness. The boundaries of a structure are usually represented in idealized forms, which are far from being real. For example, a beam that is connected to rigid supports at its ends, but free to rotate, is modelled using simply-supported (pinned) boundary conditions, which require transverse deflections and moments to be zero. However, the hole and pin assembly may have small gaps, intended to cause friction, that may introduce small deflections and/or moments at the ends. ${ }^{1}$

Many researchers have studied three-dimensional vibrations in plates. For example, Srinivas et al. derived closed form solutions for simply-supported thick plates. ${ }^{2}$ Later, Leissa and Zhang obtained solutions based on the three-dimensional elasticity theory for cantilevered parallelepipeds. ${ }^{3}$ Furthermore, Malik and Bert employed the differential quadrature method to solve free vibration in three-dimensional, thick plates with two simply-supported, opposite edges; this was done in order to reduce the problem from three- to two-dimensional, since the solution in the direction in which these plates were simplysupported was known. ${ }^{4}$ Liew et al. applied the Ritz formulation to solve the free vibration problem of thick, rectangular plates. $^{5}$

Moreover, Liew et al. and Liew and Teo applied the differential quadrature method to study the free vibration of rectangular plates. ${ }^{6,7}$ Sundara Raja Iyengar and Raman obtained the natural frequencies of thick plates with clamped and simplysupported edges through the method of initial functions. ${ }^{8,9}$
Nagino et al. applied the B-spline Ritz method to analyse the free vibration of isotropic rectangular plates. ${ }^{10}$ Civalek applied the discrete singular convolution method to study the threedimensional vibration, buckling, and bending analyses of thick plates. ${ }^{11}$ Fromme and Leissa, as well as Hutchinson and Zillimer, analysed the free vibrations of a completely stress-free rectangular parallelepiped by applying the series method. ${ }^{12,13}$ Zhou et al. applied the finite layer method to study the free vibration analysis of thick, rectangular plates with point supports. ${ }^{14}$ Houmat proposed the $h$ and $p$ version finite element method based on the pentahedral $p$-element, to investigate the free vibration of several thick plates. ${ }^{15}$

The Chebyshev spectral collocation method has been applied to modal and free vibration analyses of various types of structures, due to its high rate of convergence and accuracy. Yagci et al. used a spectral Chebyshev technique for solving linear and nonlinear beam equations, and the method was applied to the Euler-Bernoulli and Timoshenko beams. ${ }^{16}$ Lin and Jen applied the Chebyshev collocation method to laminated and non-rectangular, anisotropic plates, and compared the results with those obtained from the finite element method. ${ }^{17,18}$ Trefethen obtained the natural frequencies of a clamped square Kirchhoff plate by defining a function that satisfies the boundary conditions in the $x$ and $y$ directions. ${ }^{19}$ Luo used the Chebyshev collocation method to solve a wave propagation problem in a three-dimensional cube with all sides constrained with homogenous Dirichlet boundaries, by stacking two-dimensional $(x, y)$ slice matrices along the $z$ direction. ${ }^{20}$ Butcher et al. and Sari and Butcher studied the effects of a damaged boundary (represented by a spring with a finite stiffness) for the examples of the longitudinal vibrations of a uniform bar and the transverse vibrations of a tapered beam. ${ }^{21,22}$ For three-dimensional 